Reprod. Nutr. Dévelop., 1984, 24 (5A), 543-556.

\title{
Métabolisme intraentérocytaire et absorption par la voie sanguine des acides caprique et oléique chez le rat témoin et traité par l'actidione-cycloheximide.
}

\author{
A. BERNARD, Hélène CARLIER
}

Laboratoire de Physiologie de la Nutrition, L.A. au CNRS $n^{\circ} 273$, UER ENS. BANA (Ecole Nationale Supérieure de Biologie Appliquée à la Nutrition et à l'Alimentation), Campus Universitaire Montmuzard, 21100 Dijon, France.

Summary. Intraenterocyte metabolism and blood uptake of capric and oleic acids in control and actidione-cycloheximide-treated rats.

Intestinal absorption of capric and oleic acids from the intestinal lumen into mesenteric portal vein blood was investigated in control and actidione-cycloheximide-treated rats using the ex vivo vascular perfusion technique. The measurement of ${ }^{14} \mathrm{C}$-labeled lipids, ${ }^{14} \mathrm{CO}_{2}$ and ${ }^{14} \mathrm{C}$-labeled acid-soluble products was simultaneously carried out with blood collected at 5min intervals for $60 \mathrm{~min}$. The enterocyte catabolism of capric and oleic acids, one of which was preferentially absorbed via the blood, has been interpreted by the different processes of absorption.

The greater enterocyte catabolic activity with capric acid, compared to oleic acid, could be related to the slight affinity of FABP $Z$ for this medium-chain fatty acid and also to lack of capric acid esterification; a non-negligible fraction of this fatty acid actually disappeared from the lumen and was catabolized into the enterocyte at an early stage of its absorption.

The actidione-cycloheximide treatment affected oleic acid absorption via the blood to a greater degree than that of capric acid. But it modified both intestinal blood absorption and the catabolism of the two fatty acids in the same manner.

\section{Introduction.}

Dans la muqueuse intestinale les acides gras d'origine exogène sont l'objet de différents processus d'absorption qui permettent leur libération dans le milieu intérieur. Les acides gras à chaîne courte empruntent la voie sanguine, liés à l'albumine plasmatique. Les acides gras à longue chaîne, après réestérification et intégration des triglycérides formés dans des VLDL et chylomicrons, sont véhiculés par la lymphe (Bloom et al., 1951 ; Blomstrand, 1955 ; Borgström, 1955 ; Clément et al., 1963). La muqueuse intestinale oxyderait préférentiellement les acides gras plasmatiques d'origine endogène (Gangl et Ockner, 1975).

L'actidione-cycloheximide, inhibiteur de la synthèse protéique, diminue l'estérification intraentérocytaire de l'acide oléique, qui persiste sous forme libre (Ber- 
nard et al., 1980 ; Bernard et Carlier, 1983). Simultanément, l'absorption lymphatique des acides gras à longue chaîne, en particulier de l'acide oléique, est diminuée (Glickman et al., 1972 ; Bernard et al., 1980 ; Bernard et Carlier, 1981a). On s'attendrait à retrouver dans la veine porte cet acide gras non absorbé par la voie lymphatique, par analogie avec ce qu'on peut observer dans les conditions normales, avec l'acide caprique. C'est d'ailleurs ce que suggéraient Hyun et al. (1967) dans le cas d'un défaut d'estérification des acides gras à longue chaîne dans la muqueuse. Or, il ne semble pas, d'après des études préliminaires (BondouxRebouillat et al., 1980), que cette diminution de l'absorption lymphatique de l'acide oléique soit compensée par un passage plus important par la voie sanguine.

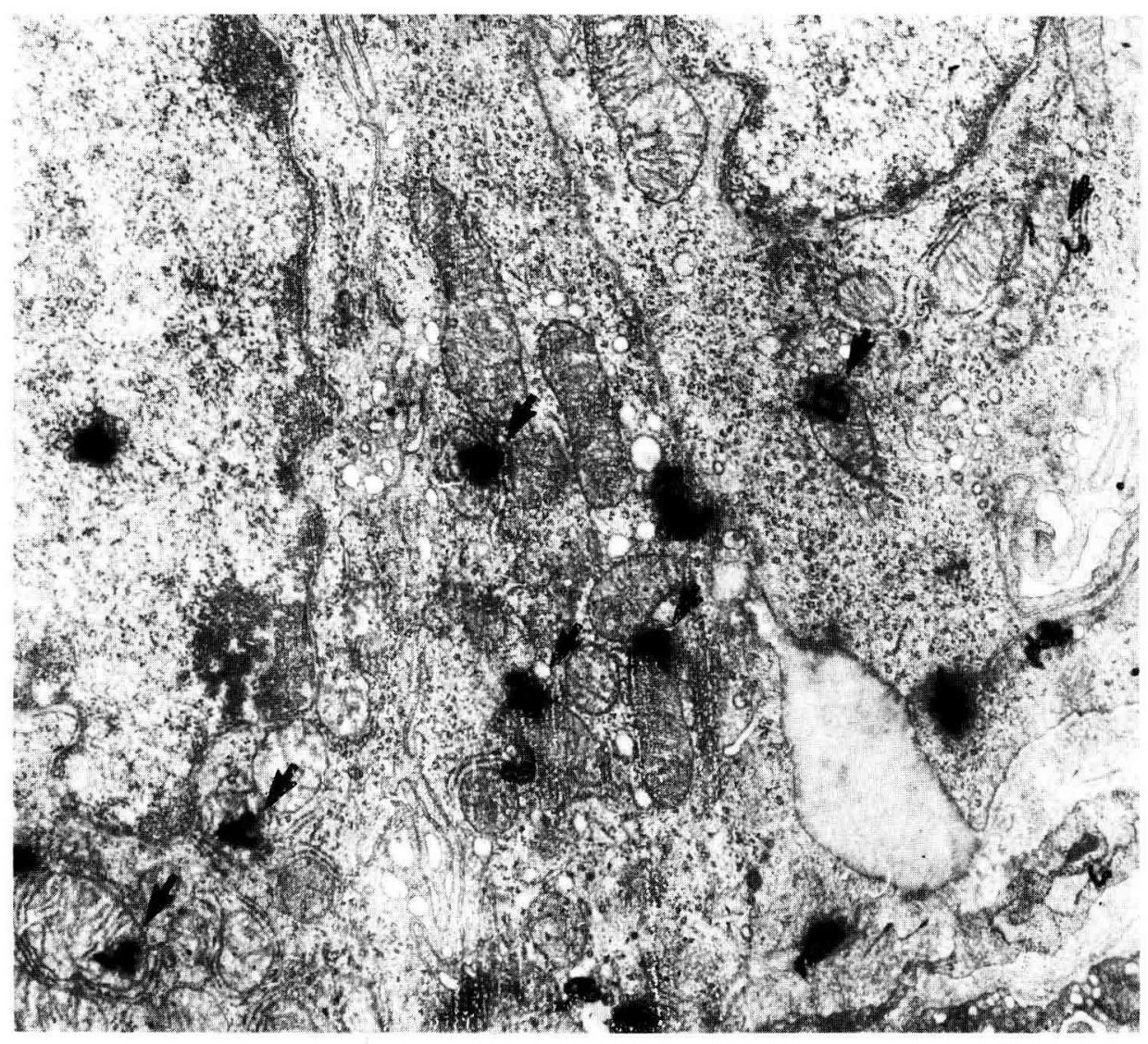

PLANCHE ।

Radioautographie en microscopie électronique, de la région infra-nucléaire d'entérocytes de jéjunum de Rat, 5 min après infusion d'acide $2-3{ }^{3} \mathrm{H}$ caprique lémulsifié en présence de monopalmitine et d'acide oléique) dans une anse intestinale isolée in situ. Des grains d'argent sont visibles au niveau des espaces intercellulaires, mais de nombreux grains d'argent sont situés sur les mitochondries $(-)$. X 12600 (Double-fixation glutaraldéhyde-tétroxyde d'osmium, émulsion nucléaire Ifford L4). 
Dans ces conditions, le catabolisme intraentérocytaire des acides gras exogènes est-il modifié ? En effet, par radioautographie en microscope électronique, nous avons remarqué au cours de l'absorption d'acide caprique titrié (PI. I) ou oléique tritié (Bernard et Carlier, 1983), aussi bien chez les rats traités que chez les rats témoins, la présence de grains d'argent au niveau ou au voisinage immédiat des mitochondries de la zone infranucléaire des entérocytes. II n'est pas possible sur les radioautographies de préciser s'il existe une réelle différence entre les lots d'animaux.

C'est pourquoi nous avons voulu évaluer in vivo, dans les conditions normales d'absorption et après traitement par la cycloheximide, le catabolisme intraentérocytaire des acides caprique et oléique dont les modalités d'absorption intestinale sont différentes. Rien n'avait été fait dans ce sens à notre connaissance, mises à part des études in vitro avec les acides octanoïque et palmitique (Porte et Entenman, 1965 ; Greenberger et al., 1965 ; Bierbach et al., 1979). Nous avons eu pour cela recours à la technique de perfusion vasculaire ex vivo adaptée d'Eloy et al. (1977) pour suivre en fonction du temps le passage par la voie sanguine des acides gras ainsi que celui de molécules reflétant leur catabolisme intraentérocytaire. L'étude sur les rats traités doit permettre de vérifier si l'inhibition de la capacité intraentérocytaire d'estérification et de formation des chylomicrons influence, en particulier, le catabolisme de l'acide oléique.

\section{Matériel et méthodes.}

Animaux: Les expériences sont réalisées sur des rats mâles adultes Wistar pesant 180 à $200 \mathrm{~g}$. Les animaux sont mis à jeun la veille de l'expérience mais gardent libre accès à l'eau de boisson.

Les animaux traités reçoivent, $4 \mathrm{~h}$ avant l'infusion intestinale du régime lipidique, une injection intrapéritonéale de $1 \mathrm{ml}$ d'une solution, dans du sérum physiologique, de $0,4 \mathrm{mg} / \mathrm{ml}$ d'actidione-cycloheximide (Actidione-Boehringer, Mannheim $\mathrm{Gmb} \mathrm{H}$ ). Les animaux témoins reçoivent une injection intrapéritonéale de même volume de sérum physiologique. La dose d'inhibiteur et le temps de l'injection avant l'expérimentation ont été choisis par référence à des travaux antérieurs (Bernard et Carlier, 1980). Le degré d'inhibition de la synthèse protéique, au moment de l'expérience, est de l'ordre de $60 \%$ (travaux non publiés).

\section{Protocole expérimental :}

- Perfusion vasculaire ex vivo. - La mise en place de la technique de perfusion vasculaire ex vivo adaptée de Eloy et al. (1977) a été rapportée en détail dans les résultats préliminaires relatifs à l'absorption par la voie sanguine de l'acide oléique (Bondoux-Rebouillat et al., 1980) et dans l'étude de l'absorption par la voie sanguine de l'acide caprique (Bernard et Carlier, 1981b).

Cette technique permet, par le cathétérisme de la veine porte, de prélever en continu la totalité du sang veineux portal mésentérique. Ainsi nous pouvons étudier l'absorption des acides gras exogènes marqués et prétendre suivre leur catabolisme intraentérocytaire, sans craindre une contamination par des molécules marquées recyclées, provenant de la circulation générale. La perte de sang est 
compensée par la perfusion dans une veine jugulaire de sang à $37^{\circ} \mathrm{C}$ de rats de même race. Le débit de perfusion est réglé de manière à équilibrer la pression artérielle du rat. Cette pression est mesurée au niveau de l'aorte abdominale infrarénale par un capteur de pression (Statham $P 23$ DB) relié à un enregistreur Racia. Dès que la pression est stable, on introduit le régime lipidique émulsionné à $37^{\circ} \mathrm{C}$ dans une anse intestinale et on collecte le sang pendant une heure par intervalle de 5 min.

- Anse intestinale isolée in situ. - On isole une anse intestinale in situ d'environ $15 \mathrm{~cm}$ entre deux canules métalliques (Collin-Gentille, $\mathrm{n}^{\circ} 18$ ) à partir du ligament de Treitz. L'anse intestinale est rincée avec $5 \mathrm{ml}$ d'une solution de Ringer à $37^{\circ} \mathrm{C}$ puis l'émulsion lipidique à $37^{\circ} \mathrm{C}$ est infusée.

- Milieux lipidiques. - Le milieu lipidique est composé, soit de $90 \mu$ moles d'acide caprique additionné de 10 à $15 \mu \mathrm{Ci}$ d'acide caprique ${ }^{14} \mathrm{C}$ marqué sur le carboxyle ( $52 \mathrm{mCi} / \mathrm{mmoles})$, soit de $30 \mu$ moles de monopalmitine, $30 \mu$ moles d'acide palmitique, $30 \mu$ moles d'acide oléique additionné de 10 à $15 \mu \mathrm{Ci}$ d'acide oléique ${ }^{14} \mathrm{C}$ marqué également sur le carboxyle $(53 \mathrm{mCi} / \mathrm{mmoles})$. Pour réaliser l'émulsion, à chaque milieu lipidique est ajouté $1,5 \mathrm{ml}$ de bile provenant d'un rat donneur. Les acides gras marqués proviennent du CEA Saclay (France). L'acide caprique a été infusé seul puisqu'il s'agit d'un acide gras à chaîne moyenne qui ne subit aucune modification chimique lors de son passage dans l'entérocyte au cours de son absorption par la voie sanguine, lié à l'albumine plasmatique. En revanche, l'acide oléique, dont l'absorption nécessite son estérification en triglycérides qui seront incorporés dans les VLDL et chylomicrons, avant leur transport par la voie lymphatique, est administré en présence d'un monoglycéride accepteur (la monopalmitine) et d'un autre acide gras (l'acide palmitique). La monopalmitine doit favoriser l'estérification par la voie des monoglycérides des acides gras prélevés. Nous avons choisi de présenter l'acide caprique seul afin d'éviter son estérification éventuelle. Nous limitons ainsi une absorption de cet acide par la voie lymphatique. Ce passage peut être facilité par les autres composants lipidiques du milieu infusé, en particulier par la présence de monoglycérides et d'acides gras à longue chaîne (publication en cours).

\section{Analyses biochimiques:}

- Extraction des lipides. - Après 60 min de contact du milieu lipidique avec la muqueuse intestinale, les lipides sont extraits selon la méthode de Delsal (1944) par du diméthoxyméthane-méthanol (4/I V/V). Les extraits sont déshydratés sous vide en présence d'alcool absolu, puis repris par du chloroforme. Sur une partie aliquote de chaque extrait, on détermine la radioactivité par scintillation liquide.

On extrait les lipides et on détermine la radioactivité de l'émulsion lipidique avant infusion et du reste de l'émulsion infusée au début de l'expérience, du contenu intestinal et de la muqueuse intestinale récupérés à la fin de l'expérience ainsi que de parties aliquotes des échantillons de sang.

- Séparation des lipides par chromatographie sur couches minces. - La méthode de Stahl (1956) permet la séparation par chromatographie sur couches minces des différentes classes de lipides des échantillons de muqueuse à la fin des expérimentations. La migration des lipides est faite par le mélange éther de 
pétrole, éther sulfurique, acide acétique, méthanol $(90 / 20 / 2 / 3 \mathrm{~V} / \mathrm{V} / \mathrm{V} / \mathrm{V})$ et l'élution après grattage est faite par $1 \mathrm{ml}$ de méthanol avant la détermination de la radioactivité.

- Dosage des catabolites. - Les bicarbonates et les produits acidosolubles proviennent de l'intégration des acides gras dans un processus oxydatif. Les bicarbonates résultent du gaz carbonique libéré par les mitochondries à partir des molécules d'acétyl CoA prises en charge par le cycle de Krebs et totalement oxydées. Les produits acidosolubles, correspondent aux acides organiques intermédiaires du cycle de Krebs, passés dans le cytoplasme.

- Dosage des bicarbonates du sang. - $1 \mathrm{ml}$ de chaque échantillon de sang est introduit, avec une pincée de saponine, dans un pot en verre. Après agitation, on ajoute $1 \mathrm{ml}$ d'acide chlorhydrique fumant. Le pot est alors fermé par un bouchon de caoutchouc sur lequel est adapté une "louche » en verre contenant de la Hyamine Packard. L'ensemble est agité et laissé incubé 20 h. On recueille ensuite la Hyamine dans un pot à comptage.

- Dosage des produits acidosolubles du sang. - On introduit $1 \mathrm{ml}$ de chaque échantilion de sang dans un tube à hémolyse avec $4 \mathrm{ml}$ d'acide perchlorique à $10 \%$. On centrifuge $10 \mathrm{~min}$ à $3000 \mathrm{~g}$. Le surnageant est filtré sur filtre millipore $0,45 \mu \mathrm{m}$. On détermine la radioactivité du filtrat.

- Mesure de la radioactivité par scintillation liquide. - Les mesures de radioactivité sont effectuées à l'aide d'un appareil à scintillation liquide Packard Prias PLD Tricarb.

Le Permafluor III Packard dilué au 1/10 dans du xylène ou du toluène est utilisé pour la détermination de la radioactivité des extraits lipidiques. L'Instagel ou le Picofluor (produits Packard) sont utilisés pour les échantillons aqueux.

\section{Résultats.}

\section{Expression des résultats.}

Dans le tableau 1 sont présentés, par rapport aux lipides infusés, les bilans globaux de l'absorption intestinale des acides caprique et oléique $60 \mathrm{~min}$ après le début de l'infusion intestinale des émulsions lipidiques. Le tableau 2 donne, par rapport aux lipides tofaux de la muqueuse intestinale, le résultat ponctuel de la radioactivité qui subsiste au niveau des acides gras libres prélevés par la muqueuse et celle intégrée dans les triglycérides de la muqueuse intestinale, 1 heure après le début de l'infusion lipidique.

Les figures 1 et 2 rendent compte de la cinétique de passage des acides caprique et oléique et de leurs catabolites dans le sang veineux portal mésentérique. Pour permettre une comparaison plus aisée des résultats, compte tenu de la dilution radio-active initiale et du marquage au niveau de la fonction carboxyle, les résultats sont exprimés en nanomoles d'acide caprique ou oléique initiales retrouvées dans le sang et en équivalents nanomoles d'acide caprique ou oléique pour les catabolites dosés. Afin d'homogénéiser la présentation des résultats et afin de comparer les cinétiques d'absorption de ces deux acides gras de longueur de chaîne différente, nous avons traduit la cinétique d'absorption de $30 \mu$ moles d'acide caprique ou de $30 \mu$ moles d'acide oléique. 


\section{TABLEAU 1}

Pourcentage de la radioactivité infusée, retrouvé dans les divers compartiments,

60 min après l'infusion intestinale dans une anse intestinale isolée in situ d'acide caprique ou oléique, marqués au ${ }^{14} \mathrm{C}$ sur le carboxyle, chez les rats témoins ou traités par l'actidione-cycloheximide sous perfusion vasculaire ex vivo.

\begin{tabular}{|c|c|c|c|c|}
\hline & \multicolumn{2}{|c|}{ Acide caprique } & \multicolumn{2}{|c|}{ Acide oléique } \\
\hline & $\begin{array}{l}\text { Rats témoins } \\
n=5\end{array}$ & $\begin{array}{c}\text { Rats traités } \\
n=3\end{array}$ & $\begin{array}{l}\text { Rats témoins } \\
n=4\end{array}$ & $\begin{array}{c}\text { Rats traités } \\
\mathrm{n}=4\end{array}$ \\
\hline I ( $\mu$ moles $)$ & $26,37(1,37)$ & $27,61 \quad(0,27)$ & $26,13 \quad(2,15)$ & $27,63(0,76)$ \\
\hline$P$ & $80,4 \quad(2,8)$ & $\left.68,2(8,5)^{*}\right)$ & $52,2(4,2)^{b}$ & $43,7 \quad(9,4)$ \\
\hline $\mathrm{M}$ & $11,5 \quad(2,3)$ & $10,4 \quad(3,9)$ & $22,6(4,2))^{a}$ & $26,8 \quad(6,3)$ \\
\hline $\mathrm{D}$ & $68,9 \quad(1,2)$ & $57,8 \quad(4,7)(* *)$ & $30,2(6,7) \mathrm{b}$ & $16,9 \quad(8,2) i * i$ \\
\hline LS & $32,5(10,0)$ & $\{3,9\}$ & $3,84(0,82) b$ & $1,37(0,61)^{(* *)}$ \\
\hline BS & $2,14 \quad(1,0)$ & $1,7 \quad(0,1)$ & $0,20(0,07) a$ & $0,15(0,04)$ \\
\hline AsS & $3,46(1,23)$ & $2,04 \quad(0,43)$ & $0,57(0,05) \mathrm{b}$ & $0,29(0,02)\left(^{* *}\right.$ \\
\hline$x$ & $29,4(12,8)$ & $21,6 \quad(7,1)$ & $21,6 \quad(9,9)$ & $14,6 \quad(8,2)$ \\
\hline
\end{tabular}

Pourcentages, par rapport à la radioactivité infusée (I) prise égale à 100, de la radioactivité prélevée par la muqueuse intestinale (P), contenue dans la muqueuse $(\mathrm{M})$ et disparve de la muqueuse $(\mathrm{D}=$ P-M), ainsi que celle retrouvée dans les lipides (LS), les bicarbonates (BS) et les catabolites acidosolubles (AsS) du sang veineux portal mésentérique.

$X$ représente le pourcentage de radioactivité non retrouvé dans le contenu, la muqueuse et le sang par rapport à la radioactivité infusée.

$n$ représente le nombre d'animaux, les valeurs sont des moyennes (écarts types).

Test de Student : action significative de l'actidione-cycloheximide au seuil de $5 \%\left({ }^{*}\right), 1 \%\left({ }^{* *}\right)$. Différence significative de l'acide oléique par rapport à l'acide caprique au seuil de $1 \%$ (a), $1 \%$ (b) chez les animaux témoins.

TABLEAU 2

Répartition de la radioactivité dans les lipides de la muqueuse intestinale.

\begin{tabular}{cccccc} 
& \multicolumn{2}{c}{ Acide caprique $\mathrm{C} 10: 0$} & & \multicolumn{2}{c}{ Acide oléique C $18: 1$} \\
\cline { 2 - 3 } & Rats témoins & Rats traités & Rats témoins & Rats traités \\
\hline $\begin{array}{c}\text { Acide gras } \\
\text { libres }\end{array}$ & $44(14)$ & $52(13)$ & $35(17)$ & $75(5)^{* *}$ \\
Triglycérides & $47(15)$ & $40(13)$ & $55(20)$ & $18(6)^{*}$ \\
\hline
\end{tabular}

Pourcentage, par rapport aux lipides totaux de la muqueuse, de la radioactivité retrouvée au niveau des acides gras libres et des triglycérides de la muqueuse intestinale de rats témoins ou traités par l'actidione-cycloheximide, $1 \mathrm{~h}$ après infusion intestinale d'acide caprique ou oléique marqués au ${ }^{14} \mathrm{C}$ sur le carboxyle. Les lipides totaux équivalent à la présence de 2,8 et $2,6 \mu$ moles d'acide caprique respectivement chez les rats témoins et traités et à celle de 5,3 et 6,9 $\mu$ moles d'acide oléique chez les rats témoins et traités par l'actidione-cycloheximide. Les valeurs rapportées sont des moyennes (écarts types) pour les lots d'animaux correspondant aux résultats consignés dans le tableau 1 et les figures 1 et 2 .

Test de Student : action significative de l'actidione-cycloheximide au seuil de $5 \%\left({ }^{*}\right), 1 \%\left({ }^{* *}\right)$. 


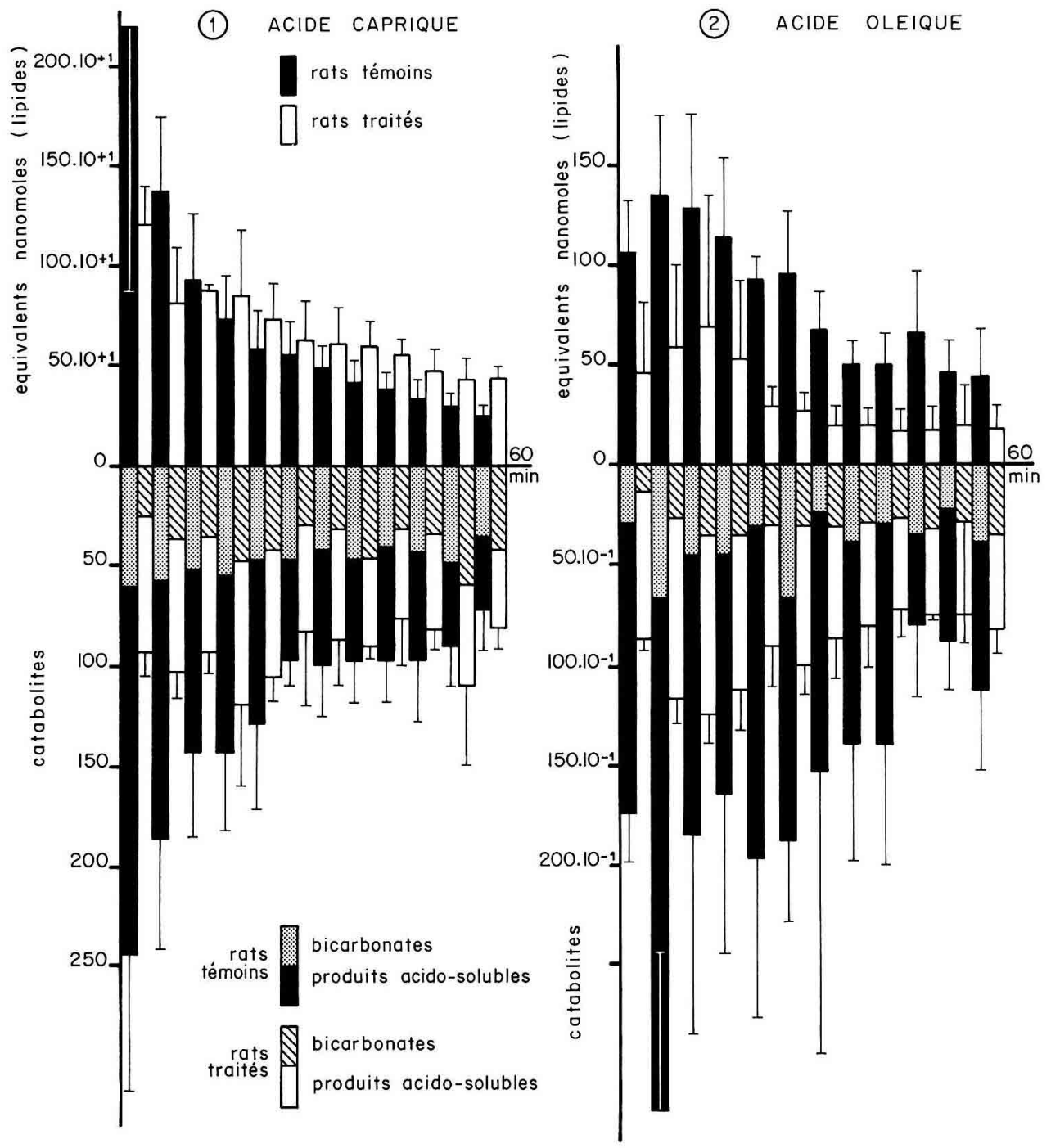

FIG. 1 et 2. - Influence des modalités d'absorption et d'un inhibiteur de la synthèse protéique sur l'absorption intestinale sanguine des acides caprique (fig. 1) et oléique (fig. 2) et leur catabolisme intraentérocytaire. L'apparition dans le sang veineux portal mésentérique des acides caprique (fig. 1) et oléique (fig. 2) marqués au ${ }^{14} \mathrm{C}$ - infusés dans une anse intestinale isolée in situ ainsi que des bicarbonates et produits acidosolubles issus de leur catabolisme intraentérocytaire est suivie par intervalles de 5 min pendant l'heure qui suit l'infusion de l'émulsion lipidique. L'expérience est faite sous perfusion vasculaire ex vivo chez les rats témoins et chez les rats traités par l'actidionecycloheximide. Les résultats sont exprimés en équivalents-nanomoles d'acide initial. 
Absorption sanguine des acides caprique et oléique.

Les résultats consignés dans ce travail sont conformes à ceux publiés pour I'acide caprique (Bernard et Carlier, 1981b) et aux résultats préliminaires obtenus avec l'acide oléique (Bondoux-Rebouillat et al., 1980).

Chez les lots d'animaux témoins la quantité d'acide caprique prélevée dans la muqueuse est très nettement supérieure à celle de l'acide oléique $180 \%$ contre $52 \%$ de l'infusé), la quantité contenue dans la muqueuse 1 heure après le début de l'infusion de l'émulsion lipidique étant moitié moindre 111,5 contre $22,6 \%$ de l'infusé). Cela conduit à une disparition de la muqueuse très supérieure avec l'acide caprique par comparaison avec l'acide oléique pour ce temps de contact et dans ces conditions opératoires (69\% contre $30 \%$ de l'infusé). Par ailleurs, l'absorption préférentielle de l'acide caprique par la voie sanguine apparaît indiscutablement, que cette absorption soit exprimée par rapport à la quantité de lipides infusée dans la lumière intestinale ou disparue de la muqueuse $132,5 \%$ contre $3,8 \%$ de l'infusé pour un flux d'entrée dans la muqueuse intestinale respectif de 80 et $52 \%$ de l'infusé ; $47,3 \%$ et $15 \%$ de la radioactivité qui disparaît de la muqueuse se retrouvent dans le sang respectivement pour l'acide caprique et l'acide oléique). En d'autres termes, exprimé en équivalents micromoles, globalement pendant l'heure de collecte du sang, pour $30 \mu$ moles de chacun des acides gras infusés, on recueille $9,74 \mu$ moles d'acide caprique $( \pm 3,0)$ et $1,15 \mu$ mole d'acide oléique $( \pm 0,24)$. La cinétique d'absorption (fig. 1$)$ met en relief une absorption maximale dans les 15 premières minutes pour l'acide caprique, avec un pic d'absorption dès les 5 premières minutes. Pour l'acide oléique, prépondérante les 15 premières minutes, l'absorption s'étale cependant plus longuement (fig. 2).

- Chez les animaux traités le tableau 1 montre que pour l'acide caprique l'absorption sanguine est peu modifiée. Par contre, comme le laissaient prévoir les résultats préliminaires, l'absorption sanguine de l'acide oléique est significativement diminuée. Conformément aux résultats illustrés dans la figure 1, l'absorption de l'acide caprique est beaucoup plus étalée ; en revanche, pour l'acide oléique (fig. 2), c'est essentiellement dans les premières $15-20$ min que se produit l'absorption.

Catabolisme des acides caprique et oléique au cours de leur absorption intestinale chez les animaux non traités.

Dans le tableau 1, les pourcentages de radioactivité, par rapport à la radioactivité infusée, portée par les produits acidosolubles et les bicarbonates retrouvés pendant l'heure de collecte du sang veineux portal mésentérique traduisent I'intensité du catabolisme au niveau de l'entérocyte. Parallèlement, les figures 1 et 2 présentent la cinétique d'apparition dans le sang de ces catabolites. Pendant I'heure d'absorption, chez les animaux témoins, 32,5\% de l'acide caprique infusé sont retrouvés dans le sang ainsi que respectivement $2,1 \%$ et $3,5 \%$ de bicarbonates et de produits acidosolubles issus de son catabolisme intraentérocytaire. La radioactivité du sang veineux portal mésentérique se répartit entre $85,3 \%$ sous forme d'acide caprique absorbé, 5,5\% sous forme bicarbonates et $9 \%$ sous forme de produits acidosolubles issus du catabolisme de cet acide gras à chaîne moyenne. 
Pendant le même temps de contact de l'émulsion lipidique à base d'acide oléique avec l'anse intestinale isolée in situ, 3,8\% de la radioactivité infusée sont retrouvés dans le sang veineux portal mésentérique, et les bicarbonates et produits acidosolubles représentent respectivement $0,2 \%$ et $0,5 \%$ de l'acide oléique infusé. La radioactivité du sang veineux portal mésentérique se répartit en $83,5 \%$ de lipides, $4,1 \%$ de bicarbonates et $12,4 \%$ de produits acidosolubles. Pour ce régime on observe pour un autre lot de rats une absorption lymphatique intestinale de 32,7\% d'acide oléique marqué infusé (Bernard et Carlier, 1981a). Chez les animaux témoins le prélèvement pour l'acide caprique $(80,4 \%$ de la radioactivité infusée) est bien supérieure à celui de l'acide oléique $(52,3 \%$ de la radioactivité infusée) et il est suivi d'une activité catabolique nettement plus importante puisque bicarbonates et produits acidosolubles représentent, respectivement pour les régimes caprique et oléique, 34,6 et $4 \%$ de la radioactivité infusée. Ainsi l'acide oléique apparaît moins catabolisé que l'acide caprique. Le pourcentage des catabolites issus de la muqueuse est pour les deux acides gras en rapport direct avec l'absorption observée par la voie sanguine.

Compte tenu de l'absorption préférentielle de l'acide caprique par la voie sanguine, la fraction catabolisée dans l'entérocyte n'est pas négligeable.

\section{Influence de l'actidione-cycloheximide sur le catabolisme intraentérocytaire des acides gras exogènes.}

Chez les animaux traités, les cinétiques de passage de l'acide caprique et de ses catabolites n'évoluent plus parallèlement, comparativement à ce qui est observé chez les animaux témoins. En revanche, ces cinétiques restent parallèles avec l'acide oléique pour les deux lots d'animaux. Pour l'acide caprique, la radioactivité retrouvée dans le sang veineux portal mésentérique est portée pour $81,7 \%$ par l'acide caprique absorbé, pour 5,1\% par les bicarbonates et pour $7,2 \%$ par les produits acidosolubles. Pour l'acide oléique, ces pourcentages respectifs sont 77,8 et $15 \%$. La valeur $X$ mentionnée dans le tableau 1 représente, comme il est indiqué, le pourcentage de radioactivité non retrouvé dans le contenu intestinal, la muqueuse intestinale, le sang veineux portal mésentérique, les bicarbonates et les produits acidosolubles, par rapport à la radioactivité infusée. Une fraction de cette valeur est à assimiler au transfert lymphatique des acides gras; quant au reste, on peut considérer que des lipides persistent dans le mésentère entre la muqueuse et les voies d'absorption. Cette valeur moyenne diminue pour les deux acides gras après le traitement, mais cette diminution n'est pas significative.

Répartition de la radioactivité au niveau des lipides de la muqueuse en fin d'expérience.

Des différences apparaissent essentiellement au niveau des acides gras libres et des triglycérides dont les pourcentages par rapport aux lipides totaux de la muqueuse sont indiqués dans le tableau 2. Ces observations indiquent la présence d'un pourcentage non négligeable de triglycérides après infusion d'acide caprique, pourcentage qui diminue après traitement. Ce fait sera discuté et explicité par 
rapport au temps expérimental de récupération de la muqueuse. Avec l'acide oléique, le traitement conduit, pour un prélèvement dans la lumière intestinale de $43 \%$ de l'acide gras infusé, à une persistance des acides gras libres dans la muqueuse (55\% contre $35 \%$ chez les rats non traités). Au contraire, on observe une diminution considérable du pourcentage de triglycérides $(18 \%$ contre $75 \%$ chez les rats non traités).

\section{Discussion.}

Absorption par la voie sanguine des acides caprique et oléique. - Il apparaît incontestablement que l'absorption intestinale de l'acide caprique se fait préférentiellement par la voie sanguine (Bloom et al., 1951 ; Borgström, 1955 ; Clément et al., 1963) surtout dans ces conditions expérimentales où aucun monoglycéride accepteur dans le régime ne favorise une estérification intraentérocytaire. Les différences observées entre les acides caprique et oléique sont à rapprocher des résultats rapportés par Greenberger et al. (1966) sur des prélèvements ponctuels de sang veineux portal mésentérique $15 \mathrm{~min}$ après infusion intestinale chez le rat d'acide octanoïque ou palmitique présentés sous forme de trioctanoïne et de tripalmitine. Nos résultats ne sont pas en contradiction avec les valeurs proposées par Hyun et al. (1967). Par ailleurs, la cinétique d'apparition des acides caprique et oléique est tout à fait conforme aux observations faites par ces derniers auteurs, pour les acides caprylique et oléique administrés seuls et dans des conditions de prélèvements ponctuels de sang veineux portal mésentérique. Cela est aussi à rapprocher des observations de Gangl et al. (1980) qui attribuent certaines différences dans le métabolisme des acides gras à une affinité différente d'une protéine de transport (FABP Z).

Dans les conditions normales, l'acide oléique, acide gras à chaîne longue, est prélevé moins rapidement dans la lumière intestinale que l'acide caprique. La proportion des lipides marqués de la muqueuse est nettement plus élevée. Ceci est compatible avec une participation active de l'entérocyte aux processus d'absorption dans le cas d'un acide gras à longue chaîne. Par ailleurs, la faible valeur du passage par la voie sanguine est révélatrice et conforme au passage préférentiel de cet acide gras à longue chaîne par la voie lymphatique.

Pour ces deux acides gras, il paraît intéressant de souligner leur absorption précoce essentiellement dès les minutes qui suivent l'infusion, suivie d'un palier correspondant à une faible absorption. Cet aspect in vivo de la cinétique d'absorption de ces deux acides gras est à rapprocher de celle observée avec l'acide palmitique par Bierbach et al. (1979) in vitro sur des entérocytes de poulet. Cette comparaison sera d'ailleurs poursuivie avec les catabolites correspondants. S'il est conforme aux données expérimentales de trouver un pourcentage élevé de triglycérides dans la muqueuse au cours de l'absorption des acides gras à longue chaîne, ceci peut surprendre au cours de l'absorption d'un acide gras à chaîne moyenne surtout lorsqu'il est administré seul. En fait, il s'agit d'un examen ponctuel de muqueuse réalisé $1 \mathrm{~h}$ après le début de l'absorption et, comme pour l'acide octanoïque (Greenberger et al., 1965 ; Carlier et Bezard, 1975), les trigly- 
cérides marqués proviennent d'acides gras à longue chaîne synthétisés par l'entérocyte à partir d'acétyl CoA issu du catabolisme de l'acide octanoïque.

La valeur $X$ non retrouvée dans les produits dosés est moindre avec l'acide oléique qu'avec l'acide caprique. Cette valeur $X$ peut être interprétée par la fraction de l'acide gras dérivé vers la voie lymphatique, dont une partie peut stagner dans les chaînes ganglionnaires.

Catabolisme intraentérocytaire et modalités d'absorption des acides gras exogènes. - Au niveau du sang veineux portal mésentérique, les bicarbonates et les produits acidosolubles dosés témoignent d'un catabolisme qui s'est effectué dans I'heure de prélèvement du sang, au niveau de la muqueuse intestinale. Veerkamp et al. (1983) soulignent, à ce propos, la nécessité de doser parallèlement les produits acidosolubles et les bicarbonates; les proportions relatives de ces deux classes de catabolites sont variables selon les tissus. Des tests effectués sur la lymphe intestinale garantissent la crédibilité de cette appréciation au niveau du sang. Lorsque, pendant I'heure de prélèvement au niveau sanguin qui suit l'infusion du régime, $2,1 \%$ et $3,5 \%$ de la radioactivité infusée est portée par les bicarbonates et les produits acidosolubles, avec un autre lot de rats munis de fistules lymphatiques, dans la lymphe recueillie pendant six heures après l'infusion du même régime caprique, seulement $0,03 \%$ et $0,09 \%$ respectivement de la radioactivité infusée sont portés par les bicarbonates et les produits acidosolubles (résultats non publiés). Ceci est en relation avec le débit sanguin infiniment plus important que le débit lymphatique conformément aux études in vitro de Greenberger et al. (1965) réalisées avec les acides octanoïque (C8:0) et palmitique (C16:0) sur des anneaux d'intestin de rat (dont respectivement 1,66 et $2,09 \%$ de la radioactivité pour l'acide octanoïque et 0,097 et $0,106 \%$ pour l'acide palmitique sont retrouvés après $30 \mathrm{~min}$ d'incubation dans les bicarbonates et les produits acidosolubles) ; nos résultats traduisent in vivo un faible catabolisme de l'acide oléique par rapport à l'acide caprique, compte tenu du pourcentage moindre d'acide prélevé par la muqueuse. Bierbach et al. (1979) mentionnent sur des entérocytes isolés de poulet un catabolisme de l'acide palmitique en bicarbonates qui atteint en une heure 15 à $20 \%$ de l'acide absorbé. L'intensité catabolique d'oxydation ne dépasse pas $5 \%$ en $1 \mathrm{~h}$ en ce qui concerne les bicarbonates mais le catabolisme global est de l'ordre de 16-18\% ; comme pour les phénomènes d'absorption, il apparaît une stabilisation des phénomènes d'oxydation (Bierbach et al., 1979). Ceci est-il à mettre en relation avec la quantité d'acide gras qui passe dans le sang portal lié à l'albumine plasmatique? En effet, si nous retenons l'hypothèse selon laquelle le catabolisme intraentérocytaire est lié à la faculté de prise en charge par la FABP Z (fatty acid-binding protein) au niveau de l'entérocyte des acides gras exogènes (Ockner et Manning, 1974 et 1976) et au degré d'activation des acides gras (Greenberger et al., 1965 et 1966 ; Gangl. et al., 1980), ces acides gras non pris en charge par la FABP $Z$ qui empruntent la voie sanguine ont court-circuité ces étapes intraentérocytaires et ont pu être captés plus facilement par les mitochondries. Cette hypothèse serait confortée par la faible quantité d'acide oléique catabolisée comparativement à la quantité prélevée.

Actidione-cycloheximide et devenir des acides gras exogènes au cours de leur absorption intestinale. - L'inhibiteur de la synthèse protéique employé se 
révèle avoir peu d'influence sur les modalités de l'absorption de l'acide gras à chaîne moyenne dont l'absorption ne requiert pas le concours d'enzymes ou de structures intraentérocytaires (Bernard et Carlier, 1981b).

L'inhibition de la synthèse protéique déprime les phénomènes de l'absorption intestinale sanguine de l'acide oléique et, parallèlement, on constate une diminution des catabolites dosés dans le sang. II semble donc qu'il $y$ ait effectivement une relation entre les acides gras non pris en charge par la FABP $Z$ et la possibilité de catabolisme des acides gras.

Bien que l'actidione-cycloheximide déprime l'absorption lymphatique des acides gras à longue chaîne, ce qui traduit ici la diminution de la valeur de $X$ chez les animaux traités, l'inhibition de la synthèse protéique ne conduit pas à un passage plus élevé par la voie sanguine (Hyun et al., 1967). Au contraire, pour l'acide oléique on observe même une légère diminution de son absorption par la voie sanguine. Avec cet acide gras, particulièrement, la tendance à la diminution de la valeur $X$ peut être la conséquence $d^{\prime}$ un passage moindre dans la voie lymphatique (Glickman et al., 1972 ; Bernard et Carlier, 1981b). En conclusion, dans nos conditions expérimentales, l'actidione-cycloheximide n'augmente ni le transfert par la voie sanguine des acides gras à longue chaîne, ni leur catabolisme intraentérocytaire.

\section{Conclusion.}

Par ponction continue de sang veineux portal mésentérique, chez le rat avec anse intestinale isolée in situ, nous avons pu observer le passage préférentiel de l'acide caprique, par rapport à l'acide oléique, par la voie sanguine. L'étude de l'absorption en fonction du temps des acides caprique et oléique, et de certains de leurs catabolites, confirme I'hypothèse d'une relation entre le catabolisme intraentérocytaire des acides gras exogènes, leur prise en charge par la FABP $Z$ et leur activation. Le traitement des animaux par l'actidione-cycloheximide n'entraîne pas, proportionnellement à l'absorption intestinale, une augmentation du catabolisme intraentérocytaire des acides caprique et oléique. II n'en reste pas moins que, par rapport à l'absorption effective, une fraction non négligeable des acides gras à chaîne moyenne est déjà catabolisée au niveau muqueuse intestinale au cours de leur absorption.

Reçu en janvier 1984 Accepté en avril 1984.

Remerciements. - Nous remercions vivement Marie-France Girardier et Marie-Claude Monnot pour leur collaboration technique. 


\section{Références}

BERNARD A., CARLIER H., 1981a. Absorption intestinale par la voie lymphatique de l'acide oléique marqué chez le rat normal et le rat traité par l'actidione-cycloheximide ou l'acétoxycycloheximide. C. R. Acad. Sci., Paris, 292, 97-100.

BERNARD A., CARLIER H., 1981b. $2-3^{3} \mathrm{H}$-decanoic acid absorption in mucosa and in portal blood in control and actidione-cycloheximide-treated rats: biochemical and electron microscope radioautographic study. Biol. Cell, 42, 115-124.

BERNARD A., CARLIER H., 1983. Biochemical and electron microscope radioautographic study of intestinal absorption of tritiated palmitic and oleic acids in control and actidionecycloheximide-treated rats. Biol. Cell, 49, 243-256.

BERNARD A., CARLIER H., CASELLI C., 1980. Etude biochimique et ultrastructurale de I'action de I'actidione-cycloheximide sur l'absorption intestinale des lipides chez le rat. J. Physiol., Paris, 76, 147-157.

BIERBACH H., HAAG G. F., HOLLDORF A. W., 1979. Uptake and metabolism of long chain fatty acids in isolated chicken intestinal epithelial cells. Digestion, 19, 392-403.

BLOMSTRAND R., 1955 . Transport form of decanoic acid ${ }^{1-14} \mathrm{C}$ in the lymph during intestinal absorption in the rat. Acta physiol. scand., 34, 67-70.

BLOOM B., CHAIKOFF I. L., REINHARDT W. O., 1951. Intestinal lymph as pathway for transport of absorbed fatty acids of different chain lengths. Amer. J. Physiol., 166, 451-455.

BONDOUX-REBOUILLAT M., BERNARD A., CARLIER H., 1980. Actidione-cycloheximide et voie d'absorption intestinale des lipides chez le rat. Reprod. Nutr. Dévelop., 20, 1325-1329.

BORGSTRÖM B., 1955. Transport form of ${ }^{14} \mathrm{C}$-decanoic acid in portal and inferior veina cava blood during absorption in the rat. Acta physiol. scand., 34, 71-74.

CARLIER H., BEZARD J., 1975. Electron microscope autoradiographic study of intestinal absorption of decanoic and octanoic acids in the rat. J. Cell Biol, 65, 383-397.

CLÉMENT G., CLEMENT J., COUREL E., KLEPPING J., BRIET S., 1963. Absorption des acides gras à chaînes moyennes et longues, 172-179. In FRAZER A. C., Biochemical problems of lipids, Elsevier, Amsterdam.

DELSAL J. L., 1944. Nouveau procédé d'extraction des lipides du sérum par le méthylal. Application aux microdosages du cholestérol total des phospholipides et des protéines. Bull. Soc. Chim. biol., 26, 99-105.

ELOY R., RAUL F., POUSSE A., MIRHOM R., ANANNA A., GRENIER J. F., 1977. Ex-vivo vascular perfusion of isolated rat small bowel. Eur. Surg. Res., 9, 96-112.

GANGL A., OCKNER R. K., 1975. Intestinal metabolism of plasma free fatty acids. Intracellular compartimentation and mechanism of control. J. clin. Invest., 55, 803-813.

GANGL A., KORNAUTH W., MLCZOCH J., SULM O., KLOSE B., 1980. Different metabolism of saturated and unsaturated long chain plasma free fatty acids by intestinal mucosa of rats Lipids, 15, 75-79.

GLICKMAN R. M., KIRSCH K., ISSELBACHER K. J., 1972. Fat absorption during inhibition of protein synthesis : studies of lymph chylomicrons. J. clin. Invest., 51, 356-363.

GREENBERGER N. J., FRANKS J. J., ISSELBACHER K. J., 1965. Metabolism of 1-C ${ }^{14}$ octanoic and 1-C ${ }^{14}$ palmitic acid by rat intestinal slices. Proc. Soc. exp. Biol. Med., 120, 468472.

GREENBERGER N. J., RODGERS J. B., ISSELBACHER K. J., 1966. Absorption of medium and long chain triglycerides: factors influencing their hydrolysis and transport. $J$. clin. Invest., 45, 217-227.

HYUN S. A., VAHOUNY G. V., TREADWELL C. R., 1967. Portal absorption of fatty acids in lymph and portal vein cannulated rats. Biochim. biophys. Acta, 137, 296-305.

OCKNER R. K., MANNING J. A., 1974. Fatty acid-binding protein in small intestine. Identification, isolation, and evidence for its role in cellular fatty acid transport. J. clin. Invest., 54, 326-338.

OCKNER R. K., MANNING J. A., 1976. Fatty acid-binding protein. Role in esterification of absorbed long chain fatty acid in rat intestine. J. clin. Invest., 58, 632-641.

OCKNER R. K., MANNING J. A., POPPENHAUSEN R. B., HO W. K. L., 1972. A binding protein for fatty acids in cytosol of intestinal mucosa, liver, myocardium, and other tissues. Science, 177, 56-58. 
PORTE D. Jr., ENTENMAN C., 1965. Fatty acid metabolism in segments of rat intestine. Am. J. Physiol., 208, 607-614.

STAHL E., SHROTER G., KRAFT G., RENZ R., 1956. Thin layer chromatography (the method affecting factors, and a few examples of application). Pharmazie, 11, 633-637.

VEERKAMP J.-M., VAN MOERKERK H.T.B., GLATZ J.F.C., VAN HINSBERGH V.W.M., 1983. Incomplete palmitate oxidation in celle-free systems of rat and human muscles. Biochim. biophys. Acta, 753, 399-410. 